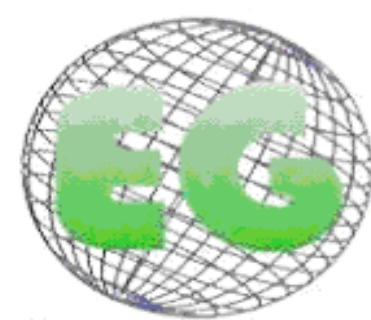

ISSN 1695-6141 N²2
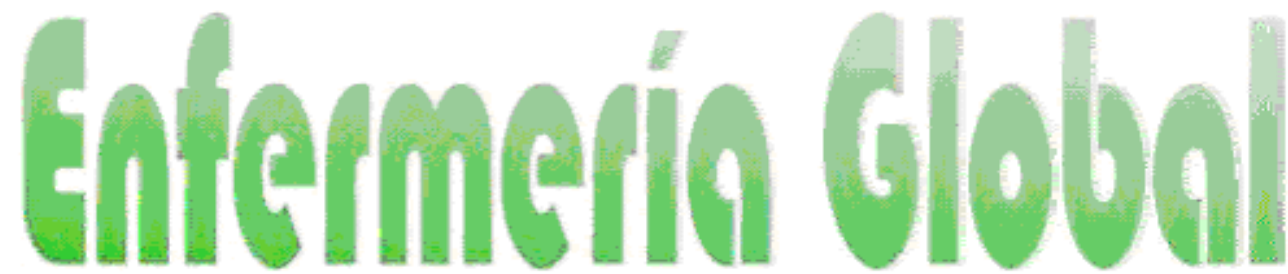

Revista electrónica trimestral de Enfermería

Abril 2011

www.um.es/egloball

CLÍNICA

\title{
FACTORES DE RIESGO QUE INCREMENTAN LA MORBIMORTALIDAD EN PACIENTES CON HEMORRAGIA DIGESTIVA ALTA
}

RISK FACTORS THAT INCREASE MORBI-MORTALITY IN GASTROINTESTINAL BLEEDING

\author{
*Rodríguez Hernández, lyemai \\ *Profesor Instructor del ISCM-H: FAC. Victoria de Girón. Cuba. \\ Palabras clave: Hemorragia digestiva alta; Factores de riesgo; Morbilidad; Mortalidad. \\ Keywords: Upper gastrointestinal bleeding; Risk factors, Morbidity; Mortality.
}

\section{RESUMEN}

La Hemorragia digestiva alta (HDA) es una emergencia médico quirúrgica frecuente que presenta una elevada morbimortalidad, constituyendo la principal urgencia en gastroenterología. El cuidado de enfermería, como el conjunto de funciones fundamentadas en la relación interpersonal y en el dominio de lo técnico-científico orientada a comprender al otro en el entorno en el que se desenvuelve, interviene en una forma importante en la prevención, la curación y la rehabilitación de la persona en el proceso salud-enfermedad.

El presente estudio tiene como objetivo identificar los factores de riesgo que incrementan la morbimortalidad y los tratamientos empleados y fundamentar la atención de enfermería. Se realiza un estudio de tipo retrospectivo con diseño de caso-control en el Hospital General Calixto García en el período Septiembre 2004 - 2008. Se llevó a cabo mediante muestreo irrestricto aleatorio, obteniéndose 150 casos, 100 para casos y 50 para controles. Los factores de riesgo asociados a morbilidad son: respecto a mayor pérdida de volumen sanguíneo, corresponde al sexo masculino total de 75 pacientes para un $78.6 \%$, el antecedente de ingestión de alcohol; para un $50 \%$ seguido del consumo de antinflamatorios no esteroides en un 33.3\%, antecedentes de úlcera péptica con $32 \%$; seguido de la diabetes Mellitus, y hipertensión arterial con un $26.6 \%$ y $23.3 \%$ respectivamente. Se manifestó clínicamente como hematemesis-melena en un $42.6 \%$ y solo hematemesis para $33.3 \%$; en cuanto a los resultados endoscópicos obtenidos la presencia de úlcera gástrica $49.3 \%$ y gastritis erosiva $24.6 \%$ fueron los más apreciados. El tratamiento médico y endoscópico fueron los más utilizados, presentando una mortalidad de $2.6 \%$.

La atención de enfermería es de vital importancia para el manejo de estos pacientes. La valoración de enfermería se basa en la observación del paciente, el interrogatorio y la determinación de las repercusiones hemodinámicas, se actuará de forma rápida y eficiente, sin precipitación, y en función del estado del paciente se establecerá la prioridad de los cuidados de enfermería. Se concluye que la gastritis erosiva y la manifestación clínica de hematemesis más melena son factores que incrementan la morbimortalidad. La disminución de la mortalidad en un $2.6 \%$ es atribuida al 
mejoramiento de las técnicas de endoscopia terapéutica y la indicación quirúrgica oportuna así como a la intervención de Enfermería.

\section{ABSTRACT}

- Gastrointestinal bleeding (UGB) is a common medical-surgical emergency with high morbidity and mortality, and represents the main emergency in gastroenterology. Nursing care, like all functions based on interpersonal relationships and the technical-scientific domain oriented towards understanding each other in the environment in which it operates, is has an important role in prevention, cure and rehabilitation of the person in the health-disease process. The present study aims to identify risk factors that increase morbidity along with the treatments used and to document nursing care. We performed a retrospective study with case-control design at the Hospital General Calixto Garcia in the period September 2004 to 2008. Simple random sampling led to 150 cases 100 for cases and 50 for controls. Risk factors associated with morbidity are: larger loss of blood volume corresponds to the total of 75 male patients, for $78.6 \%$, a history of alcohol intake, for $50 \%$ followed by the consumption of a non-steroidal anti-inflammatories 33.3\%, history of peptic ulcer $32 \%$, followed by diabetes mellitus, and hypertension with $26.6 \%$ and $23.3 \%$, respectively. This was clinically manifested as hematemesis, melena in $42.6 \%$ and $33.3 \%$ for hematemesis alone.In terms of endoscopic results, endoscopic gastric erosive gastritis, $49.3 \%$ and $24.6 \%$ were the most appreciated. The medical and endoscopic treatment were the most used, showing a mortality of $2.6 \%$. Nursing care is of vital importance for the management of these patients. Nursing assessment is based on patient observation, questioning and identification of hemodynamic effects. Action is to be quick and efficient, without haste, and depending on the patient's condition will establish the nursing care priorities. We conclude that erosive gastritis and clinical manifestation of hematemesis with melena are factors that increase morbidity and mortality. The reduction in mortality of $2.6 \%$ is attributed to improvement of endoscopic therapy and timely surgical indications and nursing intervention.

\section{INTRODUCCIÓN}

La hemorragia digestiva alta (HDA) es una emergencia médico quirúrgica frecuente que presenta una elevada morbimortalidad, a pesar de los avances terapéuticos de los últimos años, constituyendo la principal urgencia gastroenterológica. Su incidencia varía en los distintos países. Sin embargo, se estima que se presenta con una frecuencia entre 50 y 140 casos por cada cien mil habitantes con una mortalidad del 10-14\% (1-2).

Un importante número de pacientes tiene una evolución favorable y es de gran importancia identificarlos para tener un manejo adecuado de los mismos, reduciendo de esta forma los costos hospitalarios y permitiendo una rápida recuperación y reinserción a su medio (3).

Desde tiempos muy remotos se conoce la HDA como síndrome potencialmente mortal que aparece como complicación de múltiples enfermedades, constituye un reto diagnóstico y terapéutico para el médico que lo enfrenta, demanda del mismo una conducta expectante y agresiva. La HDA con sus síntomas y signos fundamentalmente es una manifestación de una enfermedad que requiere ser diagnosticada, valorada y tratada. La evolución del paciente en períodos iniciales es muy importante y con abordaje apropiado se puede manejar la situación de forma adecuada, el retardo de las decisiones o la conducta conducen a muchas complicaciones, que en ocasiones, provocan la muerte del paciente(4).

La úlcera péptica gastroduodenal, las lesiones agudas de las mucosas gástricas o duodenal y la ruptura de varices esofágicas son la etiología de HDA más frecuentemente consignada en las diferentes publicaciones (3-6). 
Desde el punto de vista de su gravedad puede abarcar desde las formas masivas agudas hasta las pérdidas sanguíneas crónicas e intermitentes, manifestándose de tres maneras: hematemesis, melena, hematoquecia, estas formas de presentación dependen de la magnitud de sangrado, evidenciándose a través de manifestaciones clínicas del compromiso hemodinámico (5-7).

Dentro de las características clínicas del paciente, la edad presenta una mortalidad de 14$35 \%$ en mayores de 60 años, a diferencia de una menor mortalidad de 4-9\% en menores de 60 años. Respecto al sexo se ha observado en pacientes con HDA que la incidencia en varones es el doble con respecto a las mujeres, siendo esta una patología que se incrementa notablemente con la edad. Otro grupo de pacientes que con mucha frecuencia padecen de gastroduodenopatías son los que ingieren fármacos anti-inflamatorios ya sean esteroideos 0 no esteroideos. Los estudios demuestran que la ingesta de grandes cantidades agudamente y en tiempo corto no desarrolla con mucha frecuencia gastropatías, pero los tratamientos crónicos y a largo plazo son los más propensos a producir irritaciones del epitelio gastroduodenal que pueden llevar a un sangrado (7-8).

Los fármacos más frecuentes son la aspirina, los anti-inflamatorios no esteroideos (AINES) y la prednisona. Le sigue el alcoholismo que es fácil entender su relación con los episodios de sangrado activo gastroduodenal, más aún, si ya se ha producido un daño hepático que condicione la producción de varices esofágicas que pueden sangrar con facilidad. Por último el antecedente de sangrado anterior es recogido por varios autores como una condicional (7-9), uno o dos de cada 10 pacientes con un sangrado actual han sufrido un episodio pasado, algunos llegan a tener un episodio anual, cada 6 meses, incluso con menor frecuencia (10). En el manejo de los factores de riesgo, lo mejor es la prevención de su aparición, su erradicación o el control; eliminando los hábitos tóxicos, los irritantes de la mucosa gastroduodenal, tratando adecuadamente las gastritis y las duodenitis, así como las úlceras.

El manejo de urgencia de la HDA con compromiso hemodinámico no depende de la lesión que la produce puesto que se dirige en todos los casos al control oportuno de la hipovolemia.

El tratamiento definitivo incluye soporte nutricional, tratamiento farmacológico eficaz en $90 \%$ de los casos, el tratamiento endoscópico si fuera necesario, con una eficacia del $80 \%$, finalmente, el tratamiento quirúrgico es el método más eficaz para detener el sangrado en úlcera péptica, así como para prevenirlo , pero su morbimortalidad hace que actualmente se reserve para casos específicos (11).

El aumento de la esperanza de vida de la población ha significado que en la actualidad esta situación se presente más frecuentemente en pacientes de edad avanzada, y en consecuencia con mayor número de patologías asociadas, por lo que cabe preguntarse ¿estamos haciendo lo correcto en nuestro medio con los pacientes con HDA?. Lla búsqueda de nuevas alternativas terapéuticas para identificar los factores de riesgo que incrementan la morbimortalidad en la comunidad y conocer la magnitud de los problemas que se relacionan a esta servirán de base para intervenciones más eficaces y oportunas. 


\section{OBJETIVOS}

\section{General:}

Identificar cuáles son los factores que incrementan la morbimortalidad de pacientes con HDA en el Hospital Universitario General "Calixto García" en el período de Septiembre 2004- 2008

\section{Específico:}

1- Caracterizar demográficamente a la población de estudio.

2- Identificar los factores de riesgo que incrementan la morbimortalidad de la HDA y su relación con la magnitud del sangrado.

3- Determinar los tratamientos empleados en pacientes con HDA.

4- Fundamentar la atención de enfermería ante esta entidad.

\section{DISEÑO METODOLOGICO}

El diseño del estudio es caso - control, de tipo retrospectivo con corte longitudinal y analítico. El universo de estudio: todos los pacientes hospitalizados con diagnóstico de hemorragia digestiva alta en el Hospital Universitario General "Calixto García" en el período de Septiembre del 2004-Septiembre 2008.

Este estudio se desarrolló mediante la obtención de una muestra probabilística con características representativas de la población de estudio y con propiedades inferenciales. Los casos y controles fueron seleccionados teniendo en cuenta la pérdida del volumen sanguíneo determinada por el grado de hemorragia digestiva alta según los cuatro grados de clasificación del Comité de Trauma del Colegio Americano de Cirugía (16). Debido a que no existe un criterio uniforme en cuanto a cómo calcular la magnitud del sangrado se utilizó un método clínico para clasificar hemodinámicamente la hemorragia digestiva siendo los controles aquellos pacientes que tuvieron pérdidas mínimas de volumen sanguíneo menores o igual a $750 \mathrm{ml}$, clasificados en el grupo I, teniendo en cuenta la situación hemodinámica de acuerdo a la gravedad: Leve- Asintomático pérdidas de $750 \mathrm{cc}=15 \%$. Mientras que los casos fueron aquellos pacientes que tuvieron pérdidas elevadas del volumen sanguíneo, mayor a $750 \mathrm{ml}$ con HDA entre los grados II y IV, manifestándose clínicamente de acuerdo a la gravedad: moderada cuando TAS mayor que $95 \mathrm{mmHg}$, F.C mayor o menor que $100 \mathrm{lpm}$ puede tener o no cambios en el ortatismo, frialdad ligera de piel y pérdidas entre $750 \mathrm{cc}-1.500 \mathrm{cc}=15-30 \%$ de volemia; grave cuando TAS menor de 95 $\mathrm{mmHg}$, F.C mayor que 120 lpm cambios ortostáticos y evidencia de hipoperfusión periférica (piel fría, pálida, sudorosa, cambios en el estado mental, oliguria, acidosis láctica) pérdidas entre 1.500-2000cc $=30-40 \%$ del volumen intravascular. $\mathrm{Y}$ masiva cuando TAS $<80$, $\mathrm{FC}>140$. Shock pérdidas $>2000 \mathrm{cc}=>40 \%$ de la volemia.

La población del estudio fue determinada mediante el reporte de pacientes hospitalizados con hemorragia digestiva alta, registrados por el departamento de estadística del Hospital, entre Septiembre 2004 - 2008, este registro contenía 100 pacientes, la información se obtuvo de las historias clínicas de cada uno de ellos. La selección del tamaño de muestra fue determinada mediante un muestreo irrestricto aleatorio sin reposición, mediante la 
estimación de la proporción poblacional. La muestra probabilística fue de 100 pacientes con hemorragia digestiva alta con un nivel de confianza de $95 \%$, un error relativo del $5 \%$ y una prevalencia del 0.35 (35\%) de pacientes con hemorragia digestiva alta con pérdidas de volumen sanguíneo de $750 \mathrm{ml}$ o más; este valor de prevalencia fue obtenida mediante una muestra aleatoria previamente seleccionada de 100 pacientes, ya que se desconocía la proporción con este diagnóstico.

Se seleccionaron aleatoriamente 100 sujetos de estudios para los casos y 50 para los controles. La selección de los sujetos fue mediante el uso de la tabla de números aleatorios.

Los criterios de inclusión establecidos para el estudio son: aquellos pacientes con diagnósticos de hemorragia digestiva alta, con historia clínica completa, hospitalizados entre los años 2004 -2008 y mayores de 18 años.

Se excluyó a los pacientes con hemorragia digestiva alta menores de 18 años debido a que presentan características diferentes a la población objeto de estudio, como es el nivel de hemoglobina y hematocrito; y sus agentes causales; así como el método para medir y clasificar el grado de hemorragia digestiva alta la cual es exclusivamente para sujetos adultos, debido a que los pacientes pediátricos tienen menor volumen sanguíneo al compararlo con los adultos (17), siendo este un posible sesgo.

Para analizar la asociación entre las variable y la magnitud del sangrado los resultados fueron sometidos a procesos estadísticos y empíricos (la encuesta - Anexo I). La encuesta fue rellenada recolectando los datos de las historias clínicas de los pacientes.

En todas las pruebas estadísticas utilizadas se prefijó un nivel de significación del 5\%. Para describir el comportamiento de todas las variables en estudio se utilizaron tablas de contingencia. Para determinar la existencia 0 no de homogeneidad en las variables cualitativas estudiadas con relación a los grupos de casos y controles se utilizó la prueba Chi-Cuadrado para variables cualitativas, rechazándose en todos los casos la hipótesis nula de homogeneidad cuando la probabilidad $p$ asociada al estadígrafo de prueba Chi-Cuadrado de Pearson resultó menor que el nivel de significación prefijado. En los casos de tablas de contingencia $2 \times 2$ se consideró entonces la corrección por continuidad de Yates más apropiada para estos casos y cuando se incumplió el requerimiento de que ninguna frecuencia esperada en base a la hipótesis nula de homogeneidad fuese menor que 5 se utilizó la prueba exacta de Fisher recomendada para estos casos. En los casos en que la prueba resultó significativa se utilizó además la razón de Productos Cruzados (odds-ratio), para identificar el número de veces en que era mayor la posibilidad de ocurrencia de la hemorragia digestiva alta con el factor de riesgo en estudio que en las no sometidas al factor, determinándose el intervalo de confianza (IC) de estos resultados.

Para todo el procesamiento de la información se utilizó el paquete profesional estadístico SPSS (Statistical Package for Social Sciences) versión 11.5.

Se empleó una PC Pentium IV con ambiente de Windows XP. Los textos se procesaron en Word XP y las tablas y gráficos se realizaron en Excel XP.

\section{RESULTADOS}

Se estudió las características de los pacientes con hemorragia digestiva alta, y se observó en la muestra estudiada una mayor frecuencia de HDA en pacientes de sexo masculino y edad mayor de 60 años (Tabla 1). 
Tabla № 1.Distribución demográfica según edad y sexo

\begin{tabular}{|l|c|c|c|l|l|l|}
\hline \multicolumn{7}{|c|}{ EDAD } \\
\hline & Casos & Controles & Total & $\%$ & $\begin{array}{c}\text { Chi } \\
\text { Cuadrado }\end{array}$ \\
\hline Mayores de 60 años & 68 & 30 & 98 & 65,3 & \\
\hline Menores de 60 años & 32 & 29 & 52 & 34,6 & \\
\hline Total & 100 & 50 & 150 & 100 & \\
\hline & & & & & \\
\hline Masculino & \multicolumn{2}{|c|}{ SEXO } & & \\
\hline Femenino & 75 & 43 & 118 & 78,6 & \\
\hline Total & 25 & 7 & 22 & 21,3 & \\
\hline
\end{tabular}

Fuente: Plantilla de encuesta

(La muestra fue de 150 casos: controles $\mathrm{N}=100$, casos $\mathrm{N}=50$ )

Los antecedentes personales más frecuentes fueron el consumo de alcohol y AINES 50.0 y $33.3 \%$ respectivamente (Tabla 2 ).

Tabla № 2

Antecedentes personales

\begin{tabular}{|l|c|c|c|c|c|c|c|c|}
\hline & Casos & $\%$ & Controles & $\%$ & total & $\%$ & $\begin{array}{c}\text { Chi } \\
\text { Cuadrado }\end{array}$ & P valor \\
\hline Alcohol & 52 & 34,6 & 23 & 15,3 & 75 & 50,00 & 0.27 & 0.603 \\
\hline AINES & 30 & 20,00 & 20 & 13,3 & 50 & 33,3 & 1.08 & 0.297 \\
\hline Corticoides & 8 & 5,3 & 3 & 2 & 11 & 7,3 & & 0.752 \\
\hline Tabaco & 10 & 6,6 & 4 & 2,6 & 14 & 9,3 & & 0.774 \\
\hline Total & 100 & 66,6 & 50 & 33,3 & 150 & 100 & & \\
\hline
\end{tabular}

Fuente: Plantilla de encuesta

(La muestra fue de 150 casos: controles $\mathrm{N}=100$, casos $\mathrm{N}=50$ )

La morbilidad de la úlcera péptica, Hipertensión arterial y Diabetes Mellitus es de 32.0\%, $23.3 \%$ y $26.6 \%$ respectivamente (Tabla 3 ), 


\begin{tabular}{|l|c|c|c|c|c|c|c|c|}
\hline & Casos & $\%$ & Controles & $\%$ & total & $\%$ & $\begin{array}{c}\text { Chi } \\
\text { Cuadrado }\end{array}$ & P \\
\hline HTA & $\mathbf{2 5}$ & $\mathbf{1 6 , 6}$ & $\mathbf{1 0}$ & $\mathbf{6 , 6}$ & $\mathbf{3 5}$ & $\mathbf{2 3 , 3}$ & 0.23 & 0.632 \\
\hline Úlcera Péptica & $\mathbf{3 0}$ & $\mathbf{2 0 , 0 0}$ & $\mathbf{1 8}$ & $\mathbf{1 2 , 0}$ & $\mathbf{4 8}$ & $\mathbf{3 2 , 0 0}$ & $\mathbf{0 . 3 1}$ & $\mathbf{0 . 5 7 7}$ \\
\hline DM & $\mathbf{2 8}$ & $\mathbf{1 8 , 6}$ & $\mathbf{1 2}$ & $\mathbf{8 , 0 0}$ & $\mathbf{4 0}$ & $\mathbf{2 6 , 6}$ & 0.11 & 0.744 \\
\hline $\begin{array}{l}\text { Hepatopatías } \\
\text { crónicas }\end{array}$ & 7 & 4,6 & 6 & 4,0 & 13 & 8,6 & & 0.359 \\
\hline $\begin{array}{l}\text { Enfermedades } \\
\text { reumáticas }\end{array}$ & 6 & 4,0 & 3 & 2,0 & 9 & 6,0 & & 1.000 \\
\hline Neoplasias & 4 & 2,6 & 1 & 0,6 & 5 & 3,3 & & 0.665 \\
\hline Total & 100 & 66,6 & 50 & 33,3 & 150 & 100 & & \\
\hline
\end{tabular}

Fuente: Plantilla de encuesta

(La muestra fue de 150 casos: controles $\mathrm{N}=100$, casos $\mathrm{N}=50$ )

La úlcera fue la lesión más frecuente en más de la mitad de los pacientes con HDA que fueron diagnosticados por endoscopia, observándose un predominio de la úlcera gástrica respecto a la úlcera duodenal, seguida de la gastritis erosiva en un $24.6 \%$ (Tabla 4), siendo mayor la posibilidad de sangrado en los pacientes con esta última

\section{Tabla № 4 Resultado examen endoscópico}

\begin{tabular}{|l|c|c|c|c|c|c|c|c|c|c|}
\hline & Casos & $\%$ & Controles & $\%$ & total & $\%$ & $\begin{array}{c}\text { Chi } \\
\text { Cuadrado }\end{array}$ & $\mathrm{P}$ & O.R & $\begin{array}{c}\text { IC 95 } \\
\mathrm{a} \%\end{array}$ \\
\hline Úlcera gástrica & $\mathbf{4 5}$ & $\mathbf{3 0}$ & $\mathbf{2 9}$ & $\mathbf{1 9 , 3}$ & $\mathbf{7 4}$ & $\mathbf{4 9 , 3}$ & 1.76 & 0.184 & & \\
\hline Úlcera duodenal & 16 & 10,6 & 12 & 8,0 & 28 & 18,6 & 0.93 & 0.335 & & \\
\hline $\begin{array}{l}\text { Gastritis } \\
\text { erosiva }\end{array}$ & $\mathbf{3 0}$ & $\mathbf{2 0 , 0}$ & $\mathbf{7}$ & $\mathbf{4 , 6}$ & $\mathbf{3 7}$ & $\mathbf{2 4 , 6}$ & 4.97 & 0.056 & 3.14 & $\begin{array}{l}113- \\
920\end{array}$ \\
\hline $\begin{array}{l}\text { Várices } \\
\text { esofagogastricas }\end{array}$ & 5 & 3,3 & 2 & 1,3 & 7 & 4,6 & & 1.000 & & \\
\hline $\begin{array}{l}\text { Síndrome } \\
\text { Mallory-Weis }\end{array}$ & 4 & 2,6 & 0 & 0 & 4 & 2,6 & & 0.302 & & \\
\hline Total & 100 & 66,6 & 50 & 33,3 & 150 & 100 & & & & \\
\hline
\end{tabular}

Fuente plantilla

de encuesta

(La muestra fue de 150 casos: controles $\mathrm{N}=100$, casos $\mathrm{N}=50$ )

No se pudo saber qué signo endoscópico de valor pronóstico (Clasificación de Forrest) fue el más frecuente debido a que no todas las historias clínicas especificaban dicha clasificación. 
La hematemesis más melena es la manifestación clínica más frecuente a razón de 4.3 veces más en los pacientes con hemorragia digestiva alta (Tabla 5).

El tratamiento médico y endoscópico se realizó en la mayoría de los pacientes. (Tabla 6).

Tabla №5 Manifestaciones clínicas

\begin{tabular}{|l|c|c|c|c|c|c|c|c|l|l|}
\hline & Casos & $\%$ & Controles & $\%$ & total & $\%$ & $\begin{array}{c}\text { Chi } \\
\text { Cuadrado }\end{array}$ & P & O.R & $\begin{array}{l}\text { IC } \\
\text { a \% }\end{array}$ \\
\hline $\begin{array}{l}\text { Hematemesis- } \\
\text { Melena }\end{array}$ & $\mathbf{5 2}$ & $\mathbf{3 4 , 6}$ & $\mathbf{1 2}$ & $\mathbf{8 , 0}$ & $\mathbf{6 4}$ & $\mathbf{4 2 , 6}$ & $\mathbf{9} .57$ & 0.002 & 3.43 & $151-788$ \\
\hline Melena & 15 & 10,0 & 12 & 8,0 & 27 & 18,0 & $\mathbf{1 . 2 7}$ & $\mathbf{0 . 2 6 0}$ & & \\
\hline Hematemesis & $\mathbf{2 8}$ & $\mathbf{1 8 , 6}$ & $\mathbf{2 2}$ & $\mathbf{1 4 , 6}$ & $\mathbf{5 0}$ & $\mathbf{3 3 , 3}$ & 3.15 & 0.076 & & \\
\hline Hematoquecia & 5 & 3,3 & 4 & 2,6 & 9 & 6 & & 0.482 & & \\
\hline Total & 100 & 66,6 & 50 & 33,3 & 150 & 100 & & & & \\
\hline
\end{tabular}

(La muestra fue de 150 casos: controles $\mathrm{N}=100$, casos $\mathrm{N}=50$ )

a intervalo de confianza $95 \%$.

Tabla № 6

Tratamientos administrados

\begin{tabular}{|l|c|c|c|c|c|c|c|c|} 
& Casos & $\%$ & Controles & $\%$ & total & $\%$ & $\begin{array}{c}\text { Chi } \\
\text { Cuadrado }\end{array}$ & $\mathrm{P}$ \\
\hline Médico & 20 & & 10 & & 30 & & & \\
\hline Médico-endoscópico & $\mathbf{4 4}$ & & $\mathbf{2 3}$ & & $\mathbf{7 0}$ & & & \\
\hline $\begin{array}{l}\text { Endoscópico- } \\
\text { quirúrgico }\end{array}$ & $\mathbf{2 6}$ & & $\mathbf{1 3}$ & & $\mathbf{3 9}$ & & & \\
\hline Médico Quirúrgico & 10 & 6,6 & 4 & 2,6 & 14 & 9,3 & & 0.775 \\
\hline Total & 100 & 66,6 & 50 & 33,3 & 150 & 100 & & \\
\hline & & & & & & & & \\
\hline
\end{tabular}

Fuente: Plantilla de encuesta

(La muestra fue de 150 casos: controles $\mathrm{N}=100$, casos $\mathrm{N}=50$ )

Se registró el fallecimiento de 4 pacientes (Tabla 7) lo que significó una mortalidad del $2.6 \%$. 


\begin{tabular}{|c|c|c|c|c|c|c|c|c|}
\hline \multirow[t]{2}{*}{ Tabla No 7} & \multicolumn{6}{|c|}{ Fallecidos } & & \\
\hline & Casos & $\%$ & Controles & $\%$ & total & $\%$ & $\begin{array}{c}\text { Chi } \\
\text { Cuadrado }\end{array}$ & $\mathrm{P}$ \\
\hline Vivos & 97 & 64,6 & 49 & 32,6 & 146 & 97,3 & & 1 \\
\hline Fallecidos & 3 & 2,0 & 1 & 0,6 & 4 & 2,6 & & 1 \\
\hline Total & 100 & 66,6 & 50 & 33,3 & 150 & 100 & & \\
\hline & & & & & & & & \\
\hline
\end{tabular}

Fuente: Plantilla de

encuesta Muestra de 150

casos: controles $\mathrm{N}=100$

casos : $\mathrm{N}=50$

\section{Intervención de enfermería ante un paciente con HDA}

Son las medidas elementales para la manutención de la vida. La valoración de enfermería se basa en la observación del paciente, el interrogatorio y la determinación de las repercusiones hemodinámicas.

Se realizó mediante:

1- Valoración de la situación hemodinámica del paciente (TA, FC), signos de perfusión periférica (temperatura, coloración de la piel y extremidades.), con ello se valorará la gravedad de la hemorragia.

Se realizó teniendo en cuenta el resultado del test de ortostatismo (donde la vigilancia estrecha de los cambios en la F.C y T/A y teniendo en cuenta los síntomas añadidos: mareos intensos, síncope más signos de shock hipovolémico, sugerían la gravedad de la hemorragia, siendo su monitorización por parte del personal de enfermería de vital importancia.

\begin{tabular}{|l|c|c|}
\hline GRAVEDAD & CLíNICA & PÉRDIDAS DE VOLEMIA \\
\hline Leve & Asintomático & Hasta 750cc=15\%. \\
\hline Moderada & $\begin{array}{c}\text { TAS }>95, \mathrm{FC}>100 \circ<100 \\
\text { Frialdad ligera de piel, } \\
\text { presión del pulso disminuida } \\
\text { Test. de ortostatismo }(-) \circ(+)\end{array}$ & Entre $750 \mathrm{cc}-1.500 \mathrm{cc}=15-30 \%$ \\
\hline Grave & $\begin{array}{c}\text { TAS }<95, \mathrm{FC}>120 \\
\text { Pulso débil, sudor, palidez } \\
\text { Test de ortostatismo(+) }\end{array}$ & Entre 1.500-2000cc=30-40\% \\
\hline
\end{tabular}


2- Comprobar que el origen de la hemorragia es digestivo y localización (la clínica del paciente ) por lo que es importante distinguir entre:

- Hematemesis: vómito de sangre fresca o restos hemáticos digeridos (borra de café) Indican HDA pero hay que descartar que no sea sangre deglutida procedente del área $O R L$ o hemoptisis.

- Melena: heces negras, brillantes, pastosas, pegajosas y malolientes. Sugiere HDA o bien HDB si el tránsito es lento. Siempre descartar que no esté tomando hierro, bismuto o que haya ingerido alimentos que tiñan las heces (regaliz, morcilla, calamares en su tinta.). Para que se produzca precisa que la sangre permanezca en el tubo digestivo al menos 8 horas.

-Hematoquecia: heces sanguinolentas. Suele indicar HDB o bien HDA si el tránsito es rápido y las pérdidas importantes ( $>1$ litro en $<1$ hora).

-Rectorragia: expulsión de sangre roja por el ano. Suele indicar hemorragia en tramos distales de colon y recto aunque puede provenir de tramos superiores si el sangrado es muy intenso y el tránsito muy rápido.

-Síntomas de hipovolemia sin manifestaciones externas de sangrado: mareo, hipotensión ortostática, taquicardia, palidez, disnea, ángor, síncope.

Se realizó mediante interrogatorio minucioso y detallado, y la exploración física al llegar el paciente.

3- Búsqueda etiológica, registrar en la historia del paciente todos los datos obtenidos.

Antecedentes personales, Historia actual, importante recoger si existe ingesta de alcohol, AINES, corticosteroides, cáusticos u otros gastroerosivos y descartar la ingesta de hierro o alimentos que puedan provocar falsas melenas.

Realizando una observación de enfermería de calidad, teniendo en cuenta el método científico de la profesión El Proceso de Atención de Enfermería.

- Se actuó de forma rápida y eficiente, pero sin precipitación, y en función del estado del paciente se estableció la prioridad de los cuidados de enfermería.

*Informar siempre al paciente ante cualquier técnica o procedimiento, contribuyendo así a disminuir su ansiedad. Colocar al paciente en posición de Trendelemburg.

*Mantener permeabilidad de las vías aéreas, canalización de dos vías venosas periféricas de calibre grueso, una para comenzar reposición de la volemia (cristaloides y coloides) y otra para obtención de muestras de sangre para estudios hemoquímicos (Hb, glucemia, BUN, iones, tiempo de Quick y pruebas cruzadas), si el paciente tiene antecedentes de cardiopatías, nefropatías o entra en shock colocar catéter para medir Presión venosa central 
*Se colocará sonda nasogástrica, si no está contraindicada. Hacer lavados con SSF y aspirar. Los lavados con suero frío con el fin de causar vasoconstricción no tienen eficacia hemostática demostrada y pueden agravar el shock por lo que no deben emplearse.

Por medio de la SNG:

1.- Identificamos y localizamos el sangrado, según el aspirado:

Sanguinolento: confirma HDA activa.

Posos de café: confirma HDA y orienta a inactividad, aunque no descarta sangrado activo a niveles más bajos.

Limpio: descarta hemorragia activa en esófago y estómago pero no en duodeno. Incluso si tiene bilis no descarta lesión sangrante postpilórica.

2.- Puede servirnos de guía si se reproduce el sangrado.

3.- Eliminamos los restos de sangre facilitando la endoscopia posterior.

\section{¿Qué hacemos con la SNG?}

- Si el aspirado es limpio, retirarla.

- Si el aspirado es en pozos de café y no se va a realizar endoscopia urgente, retirarla tras lavados.

- Si el aspirado tiene sangre fresca o posos de café y se va a realizar la endoscopia urgente, mantenerla realizando lavados y con aspiración continua hasta la realización de la endoscopia

${ }^{*}$ Control frecuente de constantes vitales, sondaje vesical y diuresis horaria, en pacientes con hemorragia masiva o hemodinámicamente inestable, para valorar perfusión tisular, si volumen urinario inferior a $0.5 \mathrm{ml} / \mathrm{Kg}$./H indica grave situación de hipovolemia. Realizar ECG para valorar signos de isquemia secundaria a hemorragia, radiografía de tórax y simple de abdomen y oxigenoterapia y gasometrías.

*Preparar al paciente para gastroscopia, debe realizarse inmediatamente si sangramiento activo o inestabilidad hemodinámica al ingreso. En el resto de los casos la endoscopia se realiza en las primeras $6 \mathrm{hrs}$., desde el inicio de la hemorragia, está contraindicada en IMA con menos de 2 semanas de evolución, compromiso respiratorio severo, coma, convulsiones, inestabilidad hemodinámica.

* Para adecuado manejo del paciente será conveniente la sedación, antes de proceder a técnicas diagnosticas y terapéutica endoscópica narcóticos y benzodiacepinas, vigilando posible depresión respiratoria o hipotensión.

*Administración de fármacos prescritos: antiácidos, antagonistas receptores $\mathrm{H} 2$, somastatina y octreotido. 
*Proporcionar medidas de comodidad al paciente (PACIENTE SEMIINCORPORADO para evitar vómitos y aspiraciones.)

*Registrar en la historia de enfermería los cuidados realizados, respuesta y evolución del paciente (12-15)

\section{DISCUSIÓN}

La presencia de determinadas condiciones que se presentan en un cuadro de HDA son llamados factores de riesgo, éstos han sido estudiados por diferentes investigadores. En el presente estudio se ha determinado que existen factores de riesgo que incrementan la morbimortalidad en los pacientes con HDA. La edad, las enfermedades asociadas y la presencia de inestabilidad hemodinámica se han considerado como factores que influyen en la morbimortalidad de los pacientes con HDA (17).

A la fecha el enfoque de la evaluación de los factores de riesgo respecto a la morbimortalidad de los pacientes con HDA ha cobrado mucha importancia, es así que grupos de investigadores en el tema han propuestos los llamados "scores" de riesgo con la finalidad de evaluar lo que en este trabajo es considerado como Factores de Riesgo (18). Entre los más importantes tenemos el score de Rockell (1996) (17) es el más conocido de todos los instrumentos de riesgo para estratificar el sangrado gastrointestinal agudo. Su objetivo primario es predecir la mortalidad del paciente. El Score de Rockell incluye tres variables no endoscópicas como son edad, shock y Comorbilidad, y dos variables endoscópicas que son el diagnóstico endoscópico y la presencia o ausencia de estigmas endoscópicos de reciente sangrado.

El score de Baylor (1993), (19) fue desarrollado para predecir la recurrencia de la hemorragia, en casos de sangrado por úlcera péptica. Este sistema de puntaje tiene tres componentes: uno pre-endoscópico basado en la edad, número de enfermedades y severidad de la enfermedad, otro endoscópico basado en zona y estigma de hemorragia y uno post-endoscopia basado en la suma de los primeros componentes. El mismo grupo de investigadores quienes usaron este score validaron prospectivamente en un estudio de cohorte en pacientes con sangrado de úlcera péptica (20-23).

Otro score es el Índice Predictivo del Centro Médico Cedars- Sinai (1996), Ilay y colaboradores (24) desarrollaron este índice basado en 4 variables que han sido previamente identificados como predictores independientes de alta temprana en una hemorragia digestiva alta aguda.

1) Hallazgos endoscópicos

2) Tiempo transcurrido desde el sangrado hasta la hospitalización

3) Estado hemodinámico (signos vitales, hematócrito, tipo de síntomas y aspirado de sonda nasogástrica

4) Comorbilidad.

En un estudio prospectivo, el grupo investigador que usó este score satisfactoriamente recomendó el alta temprana en aquellos pacientes que habían sido predichos mediante este tipo de score, por ser de bajo riesgo de efectos adversos. El score Blatchford (2000) (18) es único en dos aspectos:

1) No incluye un componente endoscópico, ni su sistema de estratificación de riesgo; 
2) La medida primaria del seguimiento es definir la necesidad de la intervención clínica para controlar el sangrado.

Este score es diferente al resto, la mayoría de ellos requieren una endoscopía temprana y la medida tradicional de las variables de mortalidad, sangrado o ambos recurrente. Los investigadores desarrollaron y validaron internamente un score para predecir cuales de los pacientes hospitalizados presentaban hemorragia digestiva alta aguda (varicosa o no varicosa), requerían tratamiento para el control del sangrado (transfusión sanguínea, intervención endoscópica o quirúrgica). En nuestro medio no se ha puesto en práctica ninguno de estos scores probablemente por la falta de implementación del uso de protocolos consensuados. El presente estudio por su carácter de retrospectivo y por no encontrar historias clínicas con datos completos para trabajar bajo los scores mencionados decide estudiar los factores de riesgo sin valoración cuantitativa de los mismos, en todo caso en el futuro los trabajos de investigación prospectivos relacionados a hemorragia digestiva deberían evaluar esta entidad bajo los sistemas de scores.

En el presente decidimos tomar como elemento clave indicador de la morbimortalidad por HDA a la situación hemodinámica del paciente, debido al hecho de que la inestabilidad en esta se acompaña de una mayor morbilidad en estos pacientes. De ahí que dirigimos nuestro trabajo a pesquisar los factores que podrían asociarse a un mayor deterioro hemodinámica del paciente y por tanto una elevada morbimortalidad. En nuestro estudio se determinó que el sexo masculino está asociado a la mayor morbilidad con un $78.6 \%$, esto es respaldado por otros estudios nacionales como el del Hospital Clínico Quirúrgico de 10 de Octubre (25), donde se observa predominio del sexo masculino sobre el femenino en una relación de 3:1. Siendo más frecuente en varones mayores de 50 años. Al igual que el estudio del hospital Militar Clínico-Quirúrgico Docente "Octavio de la Concepción y de la Pedraja de Camagüey (26-27), donde predominó los varones en un $76.5 \%$ y las edades fluctuaban entre 41 - 70 años en un 53\%. Se estratificó la edad en 60 años teniendo en cuenta que en otros estudios fue un buen parámetro comparativo para determinar la morbimortalidad (2-8). El predominio en estas edades se debe quizás al consumo de drogas ulcerógenas, como tratamiento de las múltiples afecciones que suelen ser características de estos enfermos, principalmente osteomioarticulares. En nuestro país en los meses de frío se eleva la ingestión de medicamentos gastrolesivos, por un incremento de los cuadros respiratorios y agudización de las enfermedades del SOMA, frecuente en esta etapa de la vida. En este grupo de pacientes la necesidad de cirugía por hemorragia incontrolada presenta una elevada mortalidad, situada entre $25-70 \% .(27)$.

El antecedente de consumo de alcohol (50.0\% de casos), ocupa el primer lugar similar al estudio del hospital Militar Dr. Carlos J. Finlay en el que se encontró 53.8\% (11) de pacientes con antecedente de ingesta de alcohol. El efecto deletéreo del etanol al producir daño mucoso está asociado principalmente a erosiones y hemorragia subepitelial con edema circundante, con leve a moderado incremento de las células inflamatorias de la mucosa, se postula que la injuria celular se debería a las propiedades lipofílicas inherentes del etanol, a la interrupción de la barrera mucosa gástrica o ambas, o el daño directo a los pequeños vasos sanguíneos de la mucosa.

En segundo lugar encontramos antecedentes de ingesta de AINES en $33.3 \%$ de casos, de forma similar se encontró en el estudio del hospital Militar Dr. Carlos J. Finlay con una frecuencia de 24.4\%(11).Estos resultados guardan relación con los hallazgos de autores tanto nacionales como extranjeros, aunque estudios realizados en nuestro país muestran que atendiendo al número de pacientes que se realizó esofagogastroduodenoscopia más del $60 \%$ correspondió al sexo femenino lo que se contrapone a la bibliografía consultada. Esta 
diferencia constatada está probablemente relacionada con el hecho de que son las mujeres quienes constituyen en nuestro país el mayor grupo de consumidores de AINES. (29). En cambio en un estudio realizado en el hospital Enrique Cabrera se encontró que tanto el consumo de bebidas alcohólicas y AINES estuvieron presente en $19.76 \%$ de pacientes respectivamente (28).

Los AINES constituyen uno de los grupos terapéuticos de más amplia utilización en nuestro país y el mundo, además de tener como acción principal el tratamiento del dolor, son fármacos de una gran aceptación y demanda, por parte tanto de los prescriptores como de los consumidores.

El antecedente de úlcera péptica como factor de riesgo para incrementar la morbilidad estuvo presente en más de la mitad de los pacientes con hemorragia digestiva alta (32.2\%), valores similares se encontraron en un estudio prospectivo de hemorragia digestiva alta entre 1998 - 2001 en el Hospital Enrique Cabrera (29); con una mayor morbilidad de úlcera gástrica en comparación a la úlcera duodenal.

Existen diferencias entre los casos y controles en relación a los antecedentes personales de consumo de alcohol y AINES; y a los antecedentes patológicos de úlcera péptica, HTA y Diabetes Mellitus, aunque no fueron significativas estadísticamente. Estos resultados concuerdan con otras bibliografías revisadas (17).

Con respecto a los diagnósticos mencionados de causa de HDA se encontró a la úlcera gástrica seguida de la úlcera duodenal, como primera causa de HDA $(49.3 \%$ y $18.6 \%$ respectivamente), similar resultado se encontró en el estudio del Hospital Militar Dr. Carlos J Finlay $-39.5 \%$ para ulcera gástrica- (11) y del Hospital Enrique Cabrera (39.7\% también para ulcera gástrica)(29) a diferencia de otros estudios en otros hospitales donde la causa más frecuente de hemorragia digestiva alta fue úlcera duodenal como en el Hospital Clínico Quirúrgico de 10 de Octubre (22.4\%) (25), Hospital Vladimir Ilich Lenin (servicio de cirugía) (38.3\%) (28), Hospital Territorial Docente "Dr. Mario Muñoz Monroy" Matanzas (27.6\%) (30) y otros.

Al comparar el resultado endoscópico de los grupos, la única diferencia estadísticamente significativa que obtuvimos fue en los diagnósticos de gastritis erosiva, que resultó más frecuente en el grupo de los casos (evidencias clínicas de una hemorragia mayor) que en los controles. Este resultado no concuerda con estudios revisados $(31,17)$ y podría deberse a características específicas de la muestra estudiada.

La forma de presentación como melena-hematemesis y hematemesis están presentes en 4.3 veces más en el grupo de los casos, lo cual nos indica que los pacientes con este debut de una HDA van a tener un compromiso hemodinámico mayor y peor pronóstico, por lo que hay que instituir en ellos un tratamiento más enérgico. Estos hallazgos concuerdan con la bibliografía revisada (31).

El tratamiento médico-endoscópico fue el más frecuente administrado. El tratamiento médico y la endoscopia fueron las opciones terapéuticas más utilizadas. Tanto en los casos como en los controles, no hubo diferencias significativas en los tratamientos administrados en ambos grupos, aunque el tratamiento médico- quirúrgico fue más utilizado en el grupo de casos. Se ha demostrado que el tratamiento endoscópico solo o combinado con el médico o quirúrgico disminuye la mortalidad en pacientes con HDA. 
Por otra parte, en los casos de un sangramiento severo muchas veces es necesario recurrir a la cirugía para salvar la vida del paciente, el no haber encontrado diferencias significativas en nuestro estudio en los tratamientos utilizados pudiera deberse al tamaño de la muestra, con un mayor número de casos las diferencias encontradas podrían ser mayores. Otro factor que puede haber incidido y que no se tomó en consideración porque no era objeto de estudio, es el de pacientes que pueden comenzar con una hemorragia leve, por lo que fueron clasificados en el grupo de controles y después en su evolución continuar sangrando y necesitar un tratamiento más agresivo ya sea endoscópico o quirúrgico. De ahí el hecho de que los pacientes deben ser clasificados tanto clínica como endoscópicamente para tener un criterio más exacto de su ulterior evolución y la posibilidad de resangrado .Se ha demostrado que los resultados de la endoscopia pueden vaticinar, con razonable certeza, la posibilidad de un nuevo sangrado y la evolución en la mayoría de los pacientes (31). En el presente estudio el tratamiento combinado en general fue administrado al $80 \%$ de los pacientes.

Respecto a la mortalidad se ha encontrado solo un $2.6 \%$, que es bajo, teniendo en cuenta el trabajo retrospectivo de la Dra. Nancy Jiménez (32) la mortalidad en el período de 1996 1999 fue $6.25 \%$, período en el cual no se aplicaba tratamiento endoscópico, a diferencia del periodo correspondiente a los años $2000-2006$ en que la mortalidad fue de $2.25 \%$ época en la cual ya se implementó el tratamiento endoscópico; se puede observar una diferencia significativa (de $6.25 \%$ a $2.25 \%$ ). Se aprecia con frecuencia que los algoritmos seguidos en diagnósticos y tratamientos no se aplican en tiempo y forma de acuerdo a los lineamientos recomendados para estos casos. El uso de la endoscopía terapéutica para el control de la HDA, identificación de la lesión responsable de la hemorragia y establecer el pronóstico ha revolucionado el tratamiento de este problema de salud tan difícil, por lo que es importante identificar a los pacientes donde el sangrado continua o reincide para lo cual la clasificación de Forrest y colaboradores es ideal.

Se evidenció la muerte de 4 pacientes con hemorragia digestiva alta, debido a múltiples lesiones (úlcera gástrica activa, asociada esofagitis erosiva y neoplasia gástrica), antecedentes patológicos (úlcera gástrica y enfermedad reumática) y complicación con Shock Séptico y Coagulación Intravascular Diseminada, estos pacientes solo recibieron tratamiento médico. Esto corrobora que la presencia de cuatro o más enfermedades concomitantes, se encuentra asociado a mayor mortalidad (8). En cambio en el Hospital Vladimir llich Lenin, servicio de cirugía fallecieron 3 pacientes $(3.5 \%)$ debido a hemorragia masiva, cabe resaltar que los tres pacientes tenían además alguna enfermedad subyacente (27), al igual que en otros estudios la presencia de enfermedades concomitantes ensombrece el pronóstico de los pacientes.

El resultado de la mortalidad en este estudio (2.6\%) a criterio de este autor, se debe probablemente al mejoramiento en las técnicas de endoscopía terapéutica en casos de HDA. Aunque históricamente se ha comportado de manera diferente, por ejemplo, reporte presentado por el Hospital Clínico Quirúrgico de 10 de Octubre respecto a la mortalidad de pacientes con hemorragia digestiva alta entre los años $1999-2001$ ( 25 ) que fue de 4.4\%, debido al envejecimiento progresivo de la población y al aumento de proporción de pacientes con edad avanzada con enfermedades asociadas.

\section{CONCLUSIONES}

- La HDA predomino en los hombres y en los mayores de 60 años. 
-Los factores de riesgo que incrementaron la morbi-mortalidad en este estudio:

La gastritis erosiva, dada por resultado de examen endoscópico y la melena más hematemesis como forma de presentación clínica.

-El tratamiento endoscópico y el médico fueron los más utilizados.

-La disminución de la mortalidad puede ser atribuida al mejoramiento de las técnicas de endoscopia terapéutica y la indicación quirúrgica así como a la intervención de Enfermería oportuna.

\section{REFERENCIAS BIBLIOGRÁFICAS}

1- Eisen G, Dominitz J, Faigel D et al. An annoted algorithmic approach to upper gastrointestinal bleeding. Gastrointest Endosc 2001; 53(7): 853-58.

2- Calleja J, Martínez J. Hemorragia digestiva alta no varicosa. Medicine 2000; 8 (14): 725-35.

3- IM Gralnek, VA Greater Los Angeles Healthcare System, GS Dulai, VA Greater Los Angeles Healthcare System, TT Oei, Keck-USC School of Medicine, J Gornbein, UCLA School of Medicine, Incremental Value of Endoscopy in Identifying Low-Risk Persons with Acute Upper GI Hemorrhage(UGIH)

4- F. Barranco R. Principios de urgencias. Emergencias y Cuidados críticos. Edit .UNINET:2002

5- . FEU F. Complicaciones de la enfermedad ulcerosa péptica: manifestaciones clínicas, actitudes diagnósticas e indicaciones terapéuticas. Medicine 2000; 8 (03): 128-133

6- Lázaro G. Hemorragia digestiva alta. Diagnóstico y Tratamiento. Salud Rural 2003; XX (1):1-6.

7- Lanas A, Bajador E, Serrano P, Nitro vasodilators, low-dose aspirin, other nonsteroidal anti-inflammatory drugs, and the risk of upper gastrointestinal bleeding. $\mathrm{N} \mathrm{Engl} \mathrm{J} \mathrm{Med}$ 2000; 343 (12): 834-839

8- . Hawkey C.Nonsteroidal Anti-Inflammatory Drug Gastropathy. Gastroenterology 2000; 119: 521.

9- Luis Ibáñez A .Hemorragia digestiva alta. Tomado de: http://escuela.med.puc...cl

10- Martínez A, González S A, Domínguez M. Patología Digestiva Alta en pacientes de edad avanzada con anemia ferropénica: comparación entre usuarios y no usuarios de anti-inflamatorios no esteroideos. An Med Interna (Madrid) 2001; 18(7):17-20.

11- Palomino A. Experiencias en la endoscopia de urgencia en el Sangramiento Digestivo alto. Rev. Cub Med Milit.1997; 26(2): 116-121.

12-. Cabrera G, Macedo V. Hemorragia digestiva alta. En: Salaverry García O Dir. Cirugía General. Perú: Editorial Sistema de la Biblioteca de la Facultad de Medicina de la Universidad Nacional Mayor de San Marcos; 1999

13- Vargas G. Hemorragia Digestiva. En: Vargas G, Uribe R, Núñez N, Lozano A editores. Temas escogidos en Gastroenterología. Segunda Edición. Lima- Perú: Hospital Nacional Arzobispo Loayza; 2002.p.61-85.

14- Fillipi J. Clínica y Diagnóstico de la Hemorragia digestiva alta. Manual de Patología Quirúrgica .P Univ. Católica de Chile.2002.p.74-79.

15- Calabiug M. Guías Prácticas sobre Gastroenterología (VIII). Hemorragia digestiva alta: protocolo diagnóstico - terapéutico. An Esp. Pediatr.2002; 57:466-479.

16-Manual del Curso Avanzado de Apoyo Vital en Trauma. Colegio Americano de Cirugía. Sexta Edición.1997. 
17-Rockall TA, Logan RF, Devlin HB, Northfield TC. Risk assessment after acute upper gastrointestinal haemorrhage. GUT 1996; 38:316-21

18-. Blatchford O, Murray WR, Blatchford MA. A risk score to predict need for treatment for upper gastrointestinal hemorrhage. Lancet 2000; 356:1318-21

19-Canales O., Miyagui J. Hemorragia digestiva alta en el Hospital Nacional 2 de Mayo.. Comunicaciones Libres XVI Congreso Peruano de Enfermedades Digestivas. XI Congreso Peruano de Endoscopia Digestiva. 23 - 27 Nov. 1998; Rev. Gast del Perú 59:145.

20-Del Águila G, Wong H. Hemorragia Digestiva en el Hospital II IPSS Iquitos, Julio 95 Junio 98.. Comunicaciones Libres XVI Congreso Peruano de Enfermedades Digestivas. XI Congreso Peruano de Endoscopia Digestiva. 23 - 27 Nov. 1998; Rev. Gast del Perú 60:145.

21-Saeed ZA, Winchester CB, Michalez PA, Woods KL, Graham DY. A scoring system to predict rebleeding after endoscopic therapy of nonvariceal upper gastrointestinal hemorrhagic with comparison of heat probe and ethanol injection. Am J Gastroenterology 1993;88:1842-9

22- Saeed ZA, Ramírez FC, Lleep KS, Colera, Graham DY. Prospective validation of the Baylor bleeding score for predicting the likelihood of rebreeding after endoscopic hemostasis of peptic ulcers. Gastrointest Endosc 1995; 41:561-5.

23-Digestif Dr Michel NAHON Score et formules Score de Baylor, Score de Forrest, $\underline{\text { Score de }}$ Rockall Dernier ajout - dimanche 20 janvier 2008

24-Pratto, Daniel; Salgado, Miriam; Sandtner, Cecilia; Alasino, Pablo Levin, Alberto; Wulfson, Adolfo. Identificación de los pacientes con hemorragia digestiva alta no variceal de bajo riesgo: Score HECA ${ }_{-}^{\star} 3^{\circ}$ Exposición Anual "Día del Póster en el Hospital de Emergencias "Dr. Clemente Alvarez" (HECA) del Nov/01.(http:/www.rosario.gov.ar/sitio/salud/Revista_Inv-Web/ Vol. 1y 2-Art. 8

25-Hierro González A, Jiménez Sánchez N, Sangramiento digestivo alto. Comportamiento clínico en un grupo de pacientes. ISCM-Hosp. Clínico Quirúrgico de 10 de Octubre.

Revista cubana Med V 42(4).C. Habana. Julio -Agosto 2003.

26-Escleroterapia en hemorragia digestiva alta no varicosa. "Estrategias clínicas en digestivo. Protocolos. hemorragia digestiva alta no varicosa ". www.procers.com/digest .estelm sarpm - 2004

27-Roled Martínez F, Lopez Mayedo F .Hemorragia Digestiva Alta comportamiento clínico epidemiológico. Hosp. Militar Clínico Quirúrgico Docente "Octavio de la Concepción y de la Pedraja". Archivo médico de Camagüey 2004, 8(6) ISSN 10250255

28-V.Llaudy G. Sangramientos Digestivos Altos...¿Estamos actuando correcto hoy? Servicio Cirugía Hospital Docente Provincial "V.I.Lenin". Holguín. Cuba. Rev. Brigada medica cubana, Abril 2006.

29-Patricio Ortiz, Mario Díaz *, Luis Huanquilef *, Roberto González Hemorragia Digestiva alta .Revisión de 450 casos. Departamento de Medicina Interna, facultad de Medicina Sección de Gastroenterología, Hospital Enrique Cabrera, Nov. 2005.

30-Comportamiento del sangramiento digestivo alto en pacientes endoscopiados Hosp. Territorial Docente "Dr. Mario Muñoz Monroy" Calim Matanza. Rev. Med electrónica 2006, 28 (4).

31-Harrison: Principles of internal medicine. 17th Edition; Mc Grawhill.New York. 2008 pags 257-260. 
(c) COPYRIGHT Servicio de Publicaciones - Universidad de Murcia 